\title{
One-Year Mortality Rates in US Children with End-Stage Renal Disease
}

\author{
Blanche M. Chavers ${ }^{a}$ b Julia T. Molony ${ }^{b}$ Craig A. Solid ${ }^{b}$ Michelle N. Rheault ${ }^{a}$ \\ Allan J. Collins ${ }^{b, c}$ \\ ${ }^{a}$ Department of Pediatrics, University of Minnesota Masonic Children's Hospital, ${ }^{b}$ Chronic Disease Research Group, \\ Minneapolis Medical Research Foundation, and 'Department of Medicine, University of Minnesota, Minneapolis, \\ Minn., USA
}

\section{Key Words}

Dialysis · End-stage renal disease $\cdot$ Mortality $\cdot$ Pediatrics

\begin{abstract}
Background/Aims: Few published data describe survival rates for pediatric end-stage renal disease (ESRD) patients. We aimed to describe one-year mortality rates for US pediatric ESRD patients over a 15-year period. Methods: In this retrospective cohort study, we used the US Renal Data System database to identify period-prevalent cohorts of patients aged younger than 19 for each year during the period 1995-2010. Yearly cohorts averaged approximately 1,200 maintenance dialysis patients ( $60 \%$ hemodialysis, $40 \%$ peritoneal dialysis) and 1,100 transplant recipients. Patients were followed for up to 1 year and censored at change in modality, loss to follow-up, or death. We calculated the unadjusted model-based mortality rates per time at risk, withineach cohortyear, by treatmentmodality (hemodialysis, peritoneal dialysis, transplant) and patient characteristics; percentage of deaths by cause; and overall adjusted odds of mortality by characteristics and modality. Results: Approximately $50 \%$ of patients were in the age group 15-18, $55 \%$ were male, and $45 \%$ were female. The most common causes of ESRD were congenital/reflux/obstructive causes (55\%) and glomerulonephritis (30\%). One-year mortality rates showed evidence of a decrease in the number of
\end{abstract}

peritoneal dialysis patients (6.03 per 100 patient-years, 1995; $2.43,2010 ; p=0.0263)$. Mortality rates for transplant recipients (average 0.68 per 100 patient-years) were consistently lower than the rates for all dialysis patients (average 4.36 per 100 patient-years). Conclusions: One-year mortality rates differ by treatment modality in pediatric ESRD patients.

(c) 2015 S. Karger AG, Basel

\section{Introduction}

In the United States, the incidence of pediatric endstage renal disease (ESRD) increased by $7.5 \%$, from 14.6 per million population in 1995 to 15.7 in 2010 [1]. Prevalence increased by $25.3 \%$, from 71.0 per million population in 1995 to 89.0 in 2010 . Of the 1,395 patients aged 0 to 19 years who began renal replacement therapy in 2010 , $81.4 \%$ received dialysis and $18.6 \%$ underwent kidney transplant. A US study of survival in adolescents with ESRD using United States Renal Data System (USRDS) data found improved survival rates for those undergoing kidney transplant compared with those receiving maintenance dialysis [2].

Mortality rates remain a major concern for the pediatric ESRD population. Mortality rates are higher in children undergoing kidney transplant than for children in the general population, and even higher among children

\section{KARGER 125}

(c) 2015 S. Karger AG, Base

0250-8095/15/0412-0121\$39.50/0

E-Mail karger@karger.com

www.karger.com/ajn
Blanche M. Chavers, MD

Division of Nephrology

2450 Riverside Avenue, East Building, 6th Floor, MB 678

Minneapolis, MN 55455 (USA)

E-Mail chave001@umn.edu 
receiving dialysis than for children undergoing kidney transplant [3]. A recent study reported a significant decrease in overall mortality rates over time among children initiating dialysis treatment between 1990 and 2010, but did not evaluate trends in one-year mortality rates or compare rates across ESRD treatment groups [4]. Though one-year mortality rates have decreased in US adults receiving maintenance dialysis [5], we were unable to find studies that examined one-year mortality rates over time and by treatment modality for pediatric ESRD patients. Thus, in this study, we described the trends and patterns in one-year mortality rates and risk in the pediatric ESRD population over time (1995-2010) and by treatment modality and age group.

\section{Subjects and Methods}

\section{Data Sources and Study Design}

This was a retrospective cohort study carried out using USRDS data. We created annual cohorts of period-prevalent pediatric (aged $<19$ years) ESRD patients for the period 1995-2010. Annual cohorts included patients as of January 1 and patients who were incident at any time during the year. We could not analyze incident patients separately because cohort counts were too small for stable estimates, but all adjusted results included adjustment for ESRD status (incident vs. prevalent). Eligible patients were required to have full Medicare coverage for medical and physician services. Complete Medicare claims coverage was needed to allow for censoring at changes in treatment modality (fig. 1). Patients in the dialysis cohorts may have previously undergone kidney transplant. Since new cohorts were created for each year and patients were followed for up to 1 year, patients who survived and maintained eligibility appeared in multiple cohort years. In each year, followup began on January 1 for prevalent patients and on the date of ESRD initiation for incident patients. Follow-up continued until the earliest date of death, change in renal replacement therapy, loss to follow-up, or end of the cohort year (December 31). Patients could be considered incident only once, but patients who were censored during the year due to a change in treatment modality may have been included (if eligible) in the prevalent cohort of the succeeding year under their new modality.

Patient demographic and clinical characteristics included age at the beginning of follow-up (0-4, 5-9, 10-14, 15-18 years), sex, race (white, black, other), primary cause of ESRD (glomerulonephritis, congenital/reflux/obstructive, other, unknown/missing), ESRD status (incident, prevalent), and treatment modality (hemodialysis, peritoneal dialysis, transplant). Registration on a kidney transplant waiting list (yes/no) was identified from United Network for Organ Sharing data, and comorbidity status (none, $\geq 1$, missing) from the Centers for Medicare and Medicaid (CMS) Medical Evidence Report (form CMS-2728). Cause-specific mortality (cardiovascular, infection, malignancy, missing, other) was determined from the CMS ESRD Death Notification (form CMS2746); relevant codes appear in online supplementary table S1 (for all online suppl. material, see www.karger.com/doi/10.1159/ 000380828).

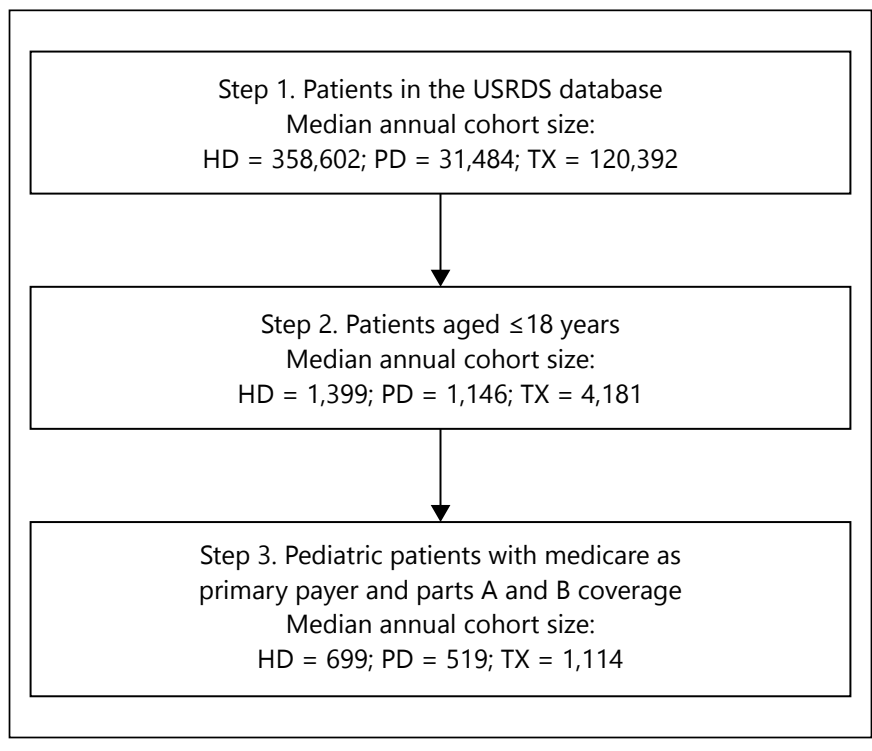

Fig. 1. Selection diagram for annual period prevalent cohorts. $\mathrm{HD}=$ Hemodialysis; $\mathrm{PD}=$ peritoneal dialysis; $\mathrm{TX}=$ transplant.

Patient characteristic variables were examined by cohort year, ESRD treatment modality, and age group. A Chi-square test for independence was used to test for differences by group; results were considered significant at the 0.05 level.

\section{Mortality Rates and Odds of Death}

The unadjusted one-year mortality rate per 100 patient-years was calculated for each annual cohort of period prevalent patients as: $100 \times$ (number of deaths)/(total time at risk, expressed in years). A model-based approach (Poisson regression) was used to estimate these rates and the corresponding 95\% confidence intervals, so weights could be incorporated into the calculations to account for differences in exposure times. Unadjusted rates were estimated separately by ESRD treatment modality, age group, and cohort year.

To study patterns in one-year mortality rates, we first considered unadjusted rates by the ESRD treatment group based on age and over time, and we used the corresponding 95\% confidence intervals to check for differences. Next, we tested for differences in the unadjusted rates between independent cohort groups. The annual cohorts were not independent by design, but by comparing 1995 versus 2010, hemodialysis versus peritoneal dialysis versus transplant (one year at a time), and younger versus older (one year at a time), we could test for significant differences in mortality rates between independent cohorts using the Poisson regression model.

Lastly, we modeled the adjusted odds of mortality using generalized estimating equations to fit a logistic regression model that included adjustment variables for all listed patient and clinical characteristics and cohort year (see table 3 for a complete list of model covariates), and adjusted for patients in multiple cohort years by including a repeated effect with an exchangeable correlation structure. All analyses were performed using SAS version 9.1 (SAS Institute). All logistic regression models were fit using SAS PROC GENMOD and included an offset variable for patient follow-up time. Results were considered significant at the 0.05 level. 
Table 1. Patient characteristics by end-stage renal disease treatment type, all years

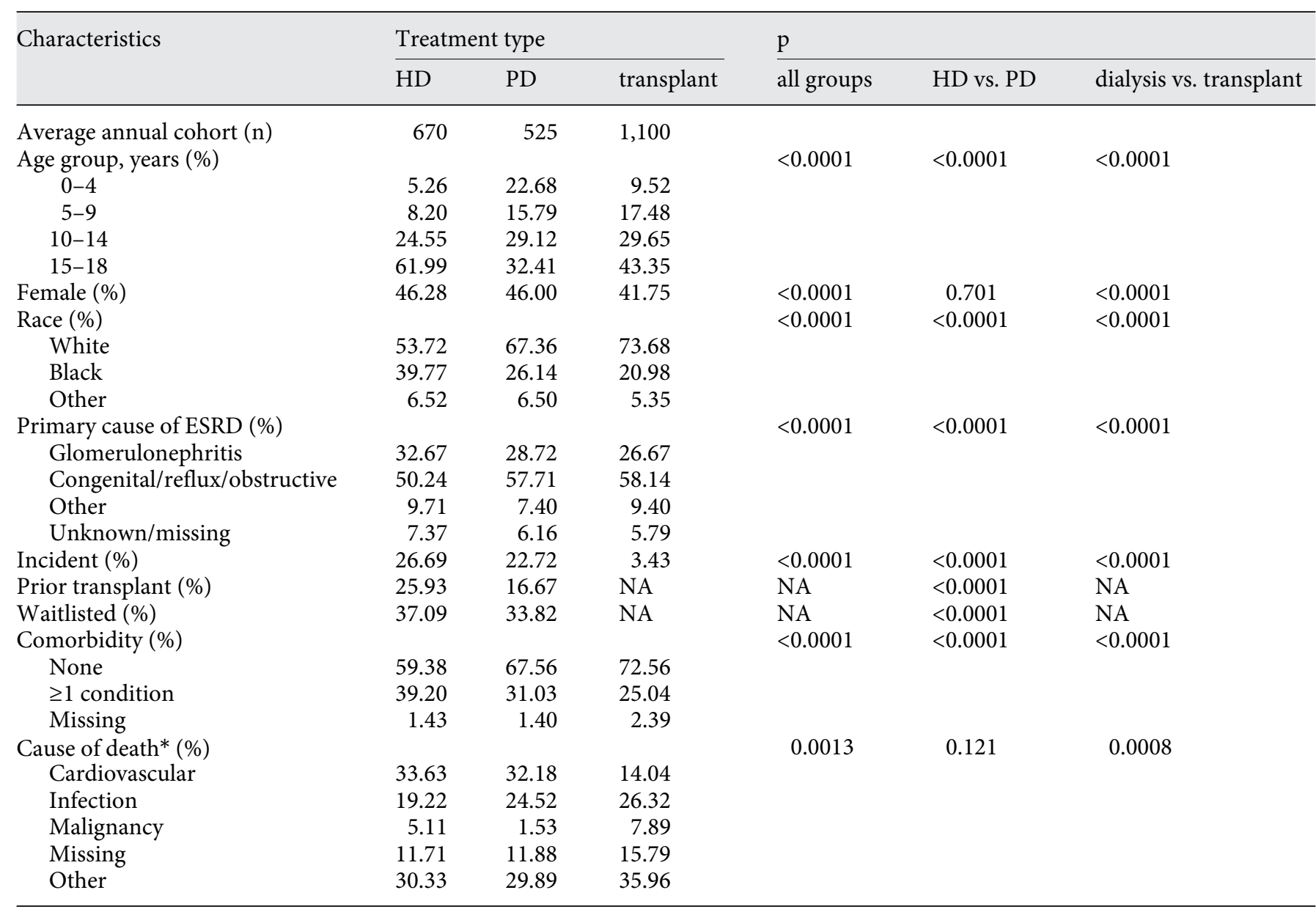

* During follow-up. ESRD = End-stage renal disease; $\mathrm{HD}=$ hemodialysis; $\mathrm{NA}=$ not applicable; $\mathrm{PD}=$ peritoneal dialysis.

\section{Results}

\section{Patient Characteristics}

Yearly cohorts included averages of approximately 1,200 maintenance dialysis patients (approximately $60 \%$ hemodialysis, $40 \%$ peritoneal dialysis; $25 \%$ incident) and 1,100 transplant recipients ( $3.5 \%$ incident). The number of annual cohort patients and demographic distributions did not change dramatically over time. Hemodialysis patients differed from peritoneal dialysis patients (table 1); they were older (average age 14.2 vs. 10.4 years) and less likely to be white (54 vs. $67 \%$ ), with lower incidence of congenital/reflux/obstructive causes as primary cause of ESRD ( 50 vs. $58 \%$ ). About $26 \%$ of hemodialysis and $17 \%$ of peritoneal dialysis patients had undergone prior kidney transplant, and a little over one-third of all dialysis patients were waitlisted for transplant. Most patients had no comorbidity, but incidence of comorbidity was higher in hemodialysis than in peritoneal dialysis or transplant patients. Transplant recipients differed from dialysis patients (table 1); they were more likely to be white (74\%) and older (10\% aged $0-4$ years vs. $23 \%$ of peritoneal dialysis patients), with less comorbidity ( $73 \%$ had none).

\section{Cause of Death}

Cause of death was not available for $12 \%$ of maintenance dialysis patients and $16 \%$ of transplant recipients. To create sufficient sample size to study the trend over time, causes of death were grouped into the categories listed in table 1. Causes included in these categories are listed in online supplementary table S1. Cardiovascular disease (average 33\%) was the most common cause of death among dialysis patients and infection (26\%) was the 
Table 2. Unadjusted 1-year mortality rates by end-stage renal disease treatment group and patient characteristics, all years

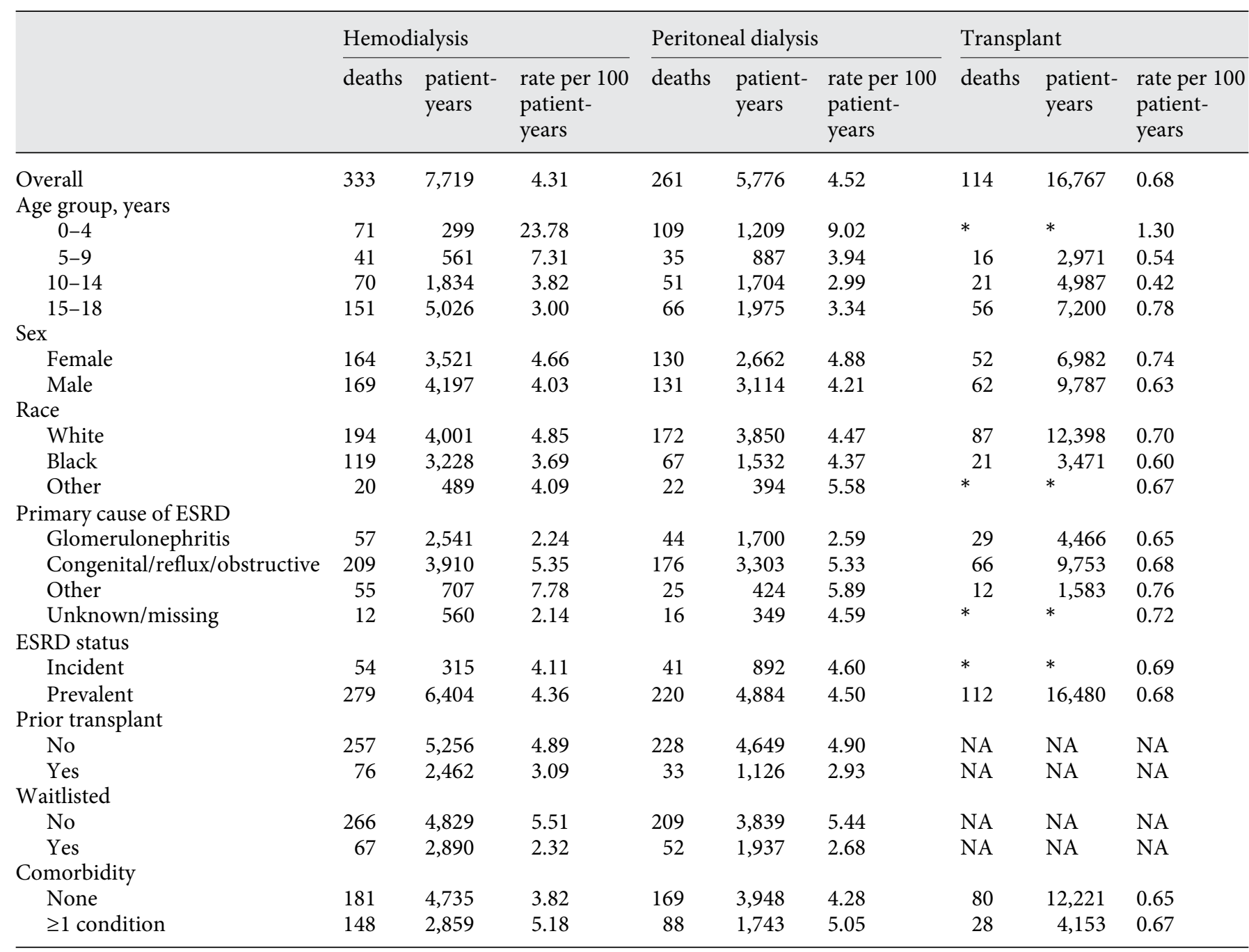

ESRD $=$ End-stage renal disease; $\mathrm{NA}=$ not applicable. ${ }^{*}$ Counts of fewer than 10 patients.

most common cause among transplant recipients (table 1). We found no change in the common causes over time, by groups of years, for any renal replacement therapy modality.

Trends in One-Year Mortality Rates

Outcomes by patient characteristics are shown in table 2 and were similar for hemodialysis, peritoneal dialysis, and transplant patients. Overall, unadjusted oneyear, model-based mortality rates per 100 patient-years were lower in transplant recipients (0.7) than in dialysis patients (4.4) (table 2). Hemodialysis and peritoneal dialysis patient mortality rates per 100 patient-years were higher for ages 0-4 and 5-9 than for other ages, for fe- male than for male patients, for white (4.9) than for black (3.7) patients, for patients with congenital/reflux/obstructive causes of ESRD than for those with glomerulonephritis, and for patients not waitlisted than for those who were waitlisted. Hemodialysis patient unadjusted one-year mortality rates were higher for white than for black patients and for patients with one or more comorbid conditions than for those with none, but were not associated with prior kidney transplant. Peritoneal dialysis patient mortality rates did not differ notably by race or comorbidity status. Transplant recipient mortality rates were higher for ages $0-4$ and 15-18 but did not differ notably by the primary cause of ESRD or comorbidity status. 


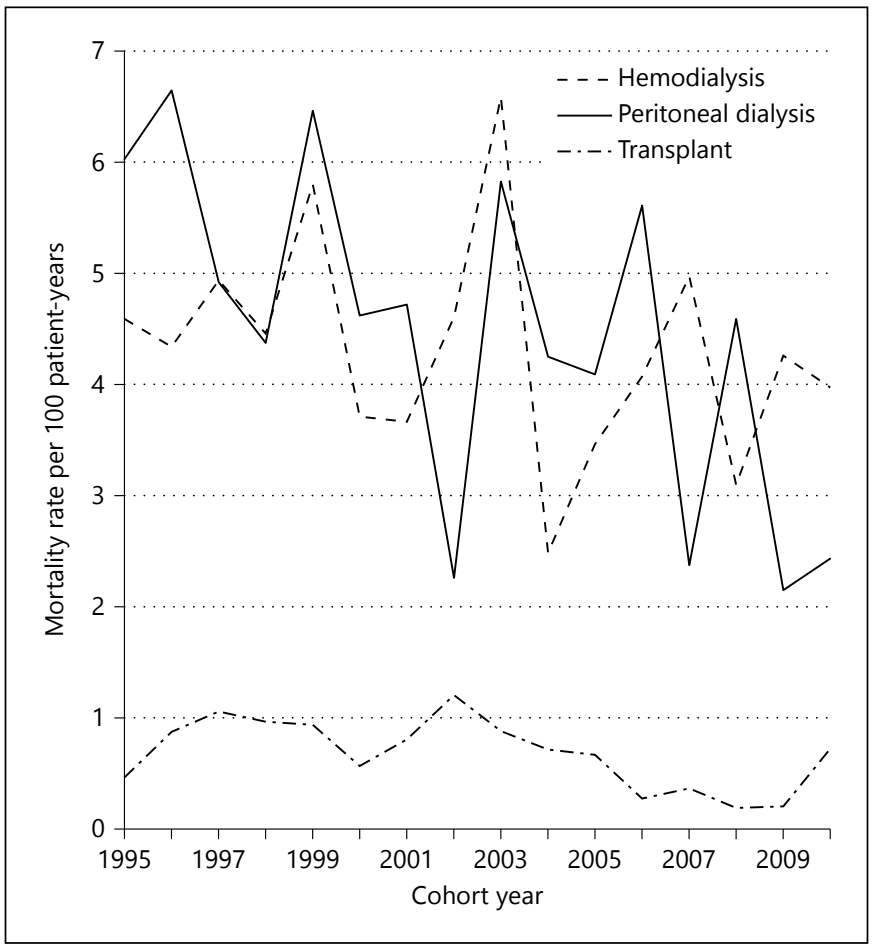

Fig. 2. Unadjusted one-year mortality rates over time, by end-stage renal disease treatment group.

Trends in unadjusted one-year mortality rates are shown in figure 2 by treatment modality. Rates fluctuated from year to year in all groups. Unlike mortality rates for hemodialysis patients, rates for peritoneal dialysis patients showed evidence of a declining trend. Three of the four lowest mortality rate years $(2002,2007,2009,2010)$ were recent; rates per 100 patient-years decreased by $60 \%$ from $1995(6.03)$ to $2010(2.43, \mathrm{p}=0.0263)$. For transplant recipients, mortality rates were significantly lower than for dialysis patients in all years, peaking at 1.2 per 100 patient-years in 2002 and 1.06 in 1997.

\section{Adjusted Odds of Mortality}

For maintenance dialysis patients, mortality risk was significantly increased for ages $0-4$ vs. $15-18$, for patients with congenital/reflux/obstructive causes of ESRD vs. glomerulonephritis, for hemodialysis patients, for prevalent patients, and for patients with one or more comorbid condition versus those with none (table 3 ). Mortality risk was significantly lower for years 2002, 2004, 2005, and 2007-2010 compared with 1995. For transplant recipients, the only significant variations in the odds of mortality occurred by age; odds were higher for ages $0-4$ and lower for ages 10-14, compared with ages 15-18.

\section{Discussion}

We studied mortality rates among cohorts of pediatric maintenance dialysis patients and kidney transplant recipients in each year from 1995 through 2010. An important finding is that one-year mortality rates for pediatric dialysis patients have not consistently declined over time, though we did observe evidence of a modest decline for peritoneal dialysis patients. Average annual mortality rates for hemodialysis and peritoneal dialysis patients were almost identical at about 4.3 per 100 patient-years (over all 15 years studied), and were consistently about 6 times higher than the average rates for transplant recipients. Using a model for the adjusted odds of mortality, we found that odds of yearly survival were better for older patients, male patients, and patients with glomerulonephritis as primary cause of ESRD compared with congenital/reflux/obstructive causes. We also found that race had no effect on the odds of survival within the cohort year.

This study provides important updates and new insights compared with prior studies evaluating mortality rates in pediatric ESRD populations. Ferris et al. [2] similarly used USRDS data from 1978 through 2002 to evaluate survival trends in incident adolescent (aged 12-19) patients with ESRD, and found improved survival for kidney transplant versus maintenance dialysis patients. Our study extends the analysis to younger patients, incident and prevalent patients, and mortality risk by dialysis modality. Our study design differs from the design of a study by Mitsnefes et al. [4] of mortality risk for children treated with dialysis. That study grouped incident patients aged younger than 21 years who started dialysis between 1990 and 2010 into 5-year calendar increments. All patients were followed until December 2010 unless censored by transplant or death. Patients in the 1990-1994 cohort were followed more than twice as long as patients in the 2005-2010 cohort. In contrast, we studied the oneyear mortality rates using annual point prevalent cohorts of patients aged younger than 19 years with up to 1 year of follow-up for each cohort. In addition, Mitsnefes et al. [4] excluded a sizable proportion of maintenance dialysis patients who had undergone prior transplant, and we included these patients.

We observed that the mortality rate was higher in peritoneal dialysis patients than among hemodialysis patients during the earlier years analyzed and lower in the later years; hence, there is evidence of decline in mortality rates for peritoneal dialysis patients, while rates for hemodialysis patients remained constant. Among pa- 
Table 3. Adjusted odds of mortality during follow-up for hemodialysis and peritoneal dialysis cohorts combined and transplant cohorts

\begin{tabular}{|c|c|c|c|c|c|}
\hline & Reference & $\begin{array}{l}\text { odds estimate } \\
(95 \% \text { CI) }\end{array}$ & $\mathrm{p}$ & $\begin{array}{l}\text { odds estimate } \\
(95 \% \text { CI) }\end{array}$ & $\mathrm{p}$ \\
\hline Age group, years & $15-18$ & & & & \\
\hline $0-4$ & & $4.71(3.7-5.99)$ & $<0.0001$ & $1.78(1.06-3)$ & 0.030 \\
\hline $5-9$ & & $1.89(1.44-2.5)$ & $<0.0001$ & $0.66(0.38-1.16)$ & 0.152 \\
\hline Male & Female & $0.77(0.65-0.91)$ & 0.002 & $0.8(0.55-1.17)$ & 0.253 \\
\hline Race group & White & & & & \\
\hline Black & & $0.98(0.81-1.18)$ & 0.817 & $0.94(0.58-1.52)$ & 0.786 \\
\hline Other & & $1.1(0.79-1.54)$ & 0.566 & $1.06(0.46-2.45)$ & 0.883 \\
\hline Primary cause of ESRD & $\begin{array}{l}\text { Congenital/reflux/ } \\
\text { obstructive }\end{array}$ & & & & \\
\hline Peritoneal & & $0.65(0.54-0.78)$ & $<0.0001$ & NA & NA \\
\hline ESRD status & Prevalent & & & & \\
\hline Incident & & $0.73(0.58-0.92)$ & 0.009 & $0.99(0.24-4.03)$ & 0.984 \\
\hline Prior transplant & No & & & & \\
\hline Yes & & $0.8(0.64-1.01)$ & 0.057 & NA & NA \\
\hline Comorbid conditions & None & & & & \\
\hline$\geq 1$ & & $1.47(1.22-1.76)$ & $<0.0001$ & $1.18(0.75-1.84)$ & 0.475 \\
\hline Missing & & $0.86(0.42-1.79)$ & 0.696 & $2.37(0.97-5.8)$ & 0.593 \\
\hline Cohort year & 1995 & & & & \\
\hline 1996 & & $1.01(0.66-1.53)$ & 0.992 & $1.91(0.65-5.6)$ & 0.241 \\
\hline 1997 & & $0.88(0.57-1.36)$ & 0.569 & $2.33(0.83-6.57)$ & 0.109 \\
\hline 2005 & & $0.58(0.36-0.92)$ & 0.022 & $1.38(0.43-4.38)$ & 0.589 \\
\hline 2006 & & $0.72(0.46-1.13)$ & 0.150 & $0.56(0.13-2.35)$ & 0.427 \\
\hline 2007 & & $0.58(0.36-0.93)$ & 0.025 & $0.73(0.19-2.76)$ & 0.644 \\
\hline 2008 & & $0.54(0.33-0.88)$ & 0.013 & $0.38(0.07-1.96)$ & 0.247 \\
\hline 2009 & & $0.46(0.28-0.75)$ & 0.002 & $0.41(0.08-2.14)$ & 0.290 \\
\hline 2010 & & $0.43(0.26-0.71)$ & 0.001 & $1.43(0.45-4.6)$ & 0.545 \\
\hline
\end{tabular}

$\mathrm{CI}=$ Confidence interval; $\mathrm{ESRD}=$ end-stage renal disease.

tients aged less than 10 , mortality rates were lower for peritoneal dialysis than for hemodialysis patients, but this difference was not apparent among patients aged 15-18 years. This is consistent with findings from Mitsnefes et al. [4], who demonstrated decreased mortality risk in peritoneal dialysis versus hemodialysis patients aged younger than 5 years, but not in children aged older than 5 years.

Patterns in mortality rates differed for hemodialysis and peritoneal dialysis patients, but an explanation was not apparent from the covariates we studied. Although hemodialysis patients were older, overall odds of mortal- 
ity were highest for younger patients. Glomerulonephritis was the leading cause of ESRD for hemodialysis patients, and was associated with lower odds of one-year mortality. Although we observed a significant difference in the racial distribution of hemodialysis and peritoneal dialysis patients, we did not find race to be a significant risk factor for mortality. Possibly, overall health differs by treatment modality, and only the healthiest ESRD patients were started on peritoneal dialysis in recent years.

Despite our findings that death rates remained constant (hemodialysis) or slightly decreased (peritoneal dialysis), mortality rates for pediatric dialysis patients remained extremely high. We found that mortality rates for pediatric dialysis patients were significantly higher than the rates for pediatric transplant recipients in all the years studied (average rates, 4.4 per 100 patient-years for dialysis patients, 0.7 for transplant recipients). The most recent National Vital Statistics Report [6] provides death rates per 100,000 patient-years for the general pediatric population in 2009; rates were $26.1,12.2,15.7$, and 53.5 per 100,000 patient-years for ages $1-4,5-9,10-14$, and 15-19, respectively. Extrapolating mortality rates from our 2009 data to the same scale gives rates of 3,394 per 100,000 patient-years for pediatric (aged 0-18) maintenance dialysis patients and 203 per 100,000 patient-years for pediatric (aged 0-19) transplant recipients. Clearly, mortality rates for pediatric ESRD patients are much higher than for the general pediatric population, and the population is growing (a 7.8\% overall increase in pediatric ESRD patients) [1]. Of concern, one-year mortality rates remained largely unchanged in the past 15 years in children undergoing maintenance hemodialysis despite technical advances in pediatric dialysis therapy over that period [7]. Kidney transplant is associated with marked reduction in mortality risk and with improved growth, neurodevelopment, and quality of life [2, 3, 8-10]. One strategy to reduce mortality in pediatric dialysis patients is earlier transplant.

Another previous study found a cardiac death rate in children with ESRD 1,000 times that in the general population [11]. In our study, cardiovascular disease was the most common cause of death for dialysis patients and the second most common cause for transplant recipients. We demonstrated that cardiovascular mortality rates remained unchanged over the past 15 years. It is a wellknown fact that there is significantly increased cardiovascular disease risk in the pediatric ESRD population compared with the general pediatric population [12]. However, at-risk children do not appear to be receiving the cardiovascular disease care that they require. Our

Mortality Rates in Children with ESRD recent study of pediatric maintenance hemodialysis patients demonstrated that only one-third had received an echocardiogram, the gold standard for diagnosis of cardiac disease [13]. Another recent study evaluated cardiovascular risk assessment in pediatric chronic kidney disease, dialysis, and kidney transplant patients at the time of transition to adult nephrology care [14]. Only $58 \%$ of cardiovascular risk factor assessments were documented, including echocardiogram, smoking assessment, family history of cardiovascular disease, lipid profile, and physical activity assessment.

This study's limitations are as follows: First, generalizability is limited to pediatric patients with Medicare as primary payer. This requirement had the greatest impact on the number of available transplant patients since many pediatric transplant patients are not fully covered by Medicare and lose Medicare eligibility after 3 years. Second, as this was an observational study using Medicare claims data, all information was limited to claims and no observed relationships could be considered causal. Third, the factors used to assign treatment modality were not available for this study and are likely nonrandom. Children who could undergo preemptive transplant were able to avoid a period of dialysis. Selection of dialysis modality for potentially sicker or high-risk patients was likely individualized to fit the needs of the patient and family. Maintenance peritoneal dialysis is the modality most commonly prescribed for infants and small children [15]. Fourth, as we chose to consider incident and prevalent patients together to increase patient counts, we could not draw conclusions about differences between these groups. The number of incident patients (and number of deaths within that group) was small enough that (1) these patients could not be analyzed separately and (2) the combined rates (incident and prevalent together) were very similar to rates for prevalent patients alone. Combining incident and prevalent patients also produced annual cohorts that were not independent, since patients who met inclusion requirements for multiple years were in multiple cohorts. However, these period-prevalent cohorts are still useful for understanding the current state of pediatric ESRD mortality. Our results describe the annual one-year mortality rates for all available patients, in each year. Fifth, our study cohort included only patients with both Part A and Part B Medicare coverage. All patients had Part A coverage. The effect of lack of Part B coverage on mortality in pediatric ESRD patients is unknown.

In conclusion, we found that one-year mortality rates among pediatric dialysis patients have not changed dra- 
matically over the past 15 years; rates for hemodialysis patients remained relatively constant and rates for peritoneal dialysis patients showed evidence of a slight decline. However, one-year mortality rates were significantly higher for pediatric maintenance dialysis patients than for pediatric kidney transplant recipients, and one-year mortality rates for all pediatric ESRD patients were much higher than for the general pediatric population. Further improvement in dialysis procedures, early transplant, and cardiovascular disease evaluation, prevention, and treatment are needed for this population.

\section{Acknowledgments}

This study was performed under Contract No. HHSN267200 715002C (National Institute of Diabetes and Digestive and Kidney Diseases, National Institutes of Health, Bethesda, Maryland). The data reported have been supplied by the US Renal Data System. The interpretation and reporting of these data are the responsibility of the authors and in no way should be seen as an official policy or interpretation of the US government. The authors have no conflicts of interest with the study's subject matter. The authors thank Chronic Disease Research Group colleagues Beth Forrest for regulatory assistance, Delaney Berrini, BS, for manuscript preparation and submission assistance, and Nan Booth, MSW, MPH, ELS, for manuscript editing.

\section{References}

1 US Renal Data System: 2012 Annual Data Report: Atlas of Chronic Kidney Disease and End-Stage Renal Disease in the United States, 2012. Bethesda, MD, National Institutes of Health, National Institute of Diabetes and Digestive and Kidney Diseases, 2012.

2 Ferris ME, Gipson DS, Kimmel PL, Eggers PW: Trends in treatment and outcomes of survival of adolescents initiating end-stage renal disease care in the United States of America. Pediatr Nephrol 2006;21:1020-1026.

3 McDonald SP, Craig JC: Long-term survival of children with end-stage renal disease. $\mathrm{N}$ Engl J Med 2004;350:2654-2662.

4 Mitsnefes MM, Laskin BL, Dahhou M, Zhang $\mathrm{X}$, Foster BJ: Mortality risk among children initially treated with dialysis for end-stage kidney disease, 1990-2010. JAMA 2013;309: 1921-1929.

5 Collins AJ, Foley RN, Gilbertson DT, Chen SC: The state of chronic kidney disease, ESRD, and morbidity and mortality in the first year of dialysis. Clin J Am Soc Nephrol 2009;4(suppl 1):S5-S11.
6 National Vital Statistics Report: Deaths: Leading Causes for 2009. Volume 61, Number 7. http://www.cdc.gov/nchs/data/nvsr/ nvsr61/nvsr61_07.pdf (accessed September 12, 2014).

7 Sebestyen JF, Warady BA: Advances in pediatric renal replacement therapy. Adv Chronic Kidney Dis 2011;18:376-383.

8 Fine RN, Martz K, Stablein D: What have 20 years of data from the North American pediatric renal transplant cooperative study taught us about growth following renal transplantation in infants, children, and adolescents with end-stage renal disease? Pediatr Nephrol 2010;25:739-746.

9 Davis ID, Chang PN, Nevins TE: Successful renal transplantation accelerates development in young uremic children. Pediatrics 1990;86:594-600.

10 Goldstein SL, Rosburg NM, Warady BA, Seikaly M, McDonald R, Limbers C, Varni JW: Pediatric end stage renal disease health-related quality of life differs by modality: a PedsQL ESRD analysis. Pediatr Nephrol 2009;24: 1553-1560.
11 Parekh RS, Carroll CE, Wolfe RA, Port FK Cardiovascular mortality in children and young adults with end-stage kidney disease. J Pediatr 2002;141:191-197.

12 Chavers BM, Li S, Collins AJ, Herzog CA: Cardiovascular disease in pediatric chronic dialysis patients. Kidney Int 2002;62:648-653.

13 Chavers BM, Solid CA, Sinaiko A, Daniels FX, Chen SC, Collins AJ, Frankenfield DL, Herzog CA: Diagnosis of cardiac disease in pediatric end-stage renal disease. Nephrol Dial Transplant 2011;26:1640-1645.

14 Hooper DK, Williams JC, Carle AC, Amaral S, Chand DH, Ferris ME, Patel HP, Licht C, Barletta GM, Zitterman V, Mitsnefes M, Patel UD: The quality of cardiovascular disease care for adolescents with kidney disease: a midwest pediatric nephrology consortium study. Pediatr Nephrol 2013;28:939-949.

15 Warady BA, Neu AM, Schaefer F: Optimal care of the infant, child, and adolescent on dialysis: 2014 update. Am J Kidney Dis 2014;64: 128-142. 